\title{
THEORETICAL SIMULATION OF TEMPERATURE DISTRIBUTION IN A GUN BARREL BASED ON THE DPL MODEL
}

\author{
Mohammad Reza Talaee, Seyed Ali Hosseinli \\ School of Railway Engineering, Iran University of Science and Technology, Tehran, Iran \\ e-mail:mrtalaee@iust.ac.ir; ali_hoseinli@rail.iust.ac.ir
}

\begin{abstract}
In this paper, an exact closed form solution is introduced for the heat conduction equation in cylindrical coordinates under consecutive inner time dependent surface heat flux by both the Fourier and dual-phase-lag (DPL) models. The solution is used to calculate the temperature distribution in a gun barrel subjected to single and consecutive shoots, and the results are compared with literature. The parametrical study is done using the analytical solution to show the effect of shooting frequency which leads to different heat power from each fire shoot and temperature distribution. The result shows good ability of analytical solution for estimation of temperature distribution in the gun barrel, especially under consecutive shoots in which unexpected incidents such as barrel melting is so probable. The closed form solution can be applied for verification of other numerical works in this area.
\end{abstract}

Keywords: DPL model, closed form solution, gun barrel, exponential decaying heat flux

\section{Introduction}

According to literature, the classical Fourier theory can estimate an acceptable thermal distribution in most engineering materials but the thermal propagation velocity in this model is infinite, thus in some applications such as great heat flux on a surface or small time of interaction this model must be improved. Determination of temperature distribution in a gun barrel that is exposed to great heat flux in a short period is one of the mentioned phenomena. At first, the non-Fourier heat flux equation as a modified model was presented by Cattaneo (1958), Vernotte (1961) (C-V relation) as a single phase model, and Tzou $(1995,1997)$ proposed a new concept of dual-phase-lag (DPL) model in which the heat flux and temperature gradient could be simulated with lag times. This model is appropriate for thermal analysis of metals (Tzou, 1995, 1997) and is used to analysis heat transfer in some relative applications (Afrin et al., 2014; Ghazanfarian and Abbassi, 2009, 2012; Ghazanfarian and Shomali, 2012; Han et al., 2006; Liu and Chen, 2010).

Considering a finite speed for thermal propagation and phonon-electron interactions in metals, the dual-phase-lag heat conduction model is

$$
q+\tau_{q} \frac{\partial q}{\partial t}=-k\left(\nabla T+\tau_{T} \frac{\partial}{\partial t} \nabla T\right)
$$

where $T$ is temperature, $q$ is heat flux, $k$ is thermal conductivity, $\tau_{q}$ shows the time lag of heat flux and $\tau_{T}$ is the time lag of the temperature gradient with constant magnitudes about picosecond and a common specific ratio of $\tau_{T} / \tau_{q}=100$ for metals (Chandrasekharaiah, 1998).

The dual-phase-lag heat conduction equation is obtained using the above constitutive relation and energy equation as

$$
\tau_{q} \frac{\partial^{2} T}{\partial t^{2}}+\frac{\partial T}{\partial t}=\alpha\left(\nabla^{2} T+\tau_{T} \frac{\partial}{\partial t} \nabla^{2} T\right)
$$


where $\alpha=k /(\rho C)$ is thermal diffusivity, $\rho$ is density and $C$ is specific heat of the material. This equation transforms to the $\mathrm{C}-\mathrm{V}$ Non-Fourier heat conduction model when $\tau_{T}=0$ and reduces to the classical Fourier heat conduction if $\tau_{T}=\tau_{q}=0$.

Some engineering problems in cylindrical coordinates are solved by a non-Fourier model. Torabi and Saedodin (2011) studied the temperature distribution in cylindrical coordinates numerically and analytically and reported that for some cases in hyperbolic heat conduction, up to $40 \%$ error occurred in the numerical method. Mishra and Sahai (2012) implemented the lattice Boltzmann method for analysis of hyperbolic heat conduction in spherical and cylindrical coordinates.

Gheitaghy and Talaee (2013) solved the hyperbolic and parabolic heat conduction by an electrical simulation method. Talaee et al. (2014) presented an exact analytical solution for non-Fourier thermal stress in a cylindrical shell considering $\mathrm{C}-\mathrm{V}$ relation under a periodic boundary condition. Saedodin and Barforoush (2017) solved the hyperbolic heat conduction equation in a typical cylinder under special heat flux and found that an increase of the Vernotte number led to delay in sense of thermal wave in a specific point.

Also the DPL method is used for analysis of thermal phenomena by some researchers. Akbarzadeh and Chen (2012) investigated the transient heat conduction in a functionally graded cylindrical panel based on DPL theory and reported the ability of this model for analysis of parabolic and hyperbolic heat conduction. In addition, they found that an increase of the phase lag led to a decrease of the speed of thermal wave along with growth in amplitude of the transient temperature. Torabi and Zhang (2014) investigated the temperature distribution of a cylindrical geometry subjected to two types of continuously and exponential pulsed heat flux boundary conditions by semi-analytical and numerical methods.

The inner surface of gun barrel is exposed to high heat load due to hot species of combustion products that must be calculated in design of an automatic gun. This phenomenon will be more critical and can threaten the shooter by self-ignition or melting due to temperature rise in the automatic gun in the continuous fire mode. In addition, change in the gun caliber due to high temperature can reduce the shooting accuracy. Therefore, for proper thermal design of the gun barrel, some research studies are done to determine the temperature of the barrel at different conditions.

The classical Fourier law is used as a practical tool to analyze most heat transfer phenomena such as thermal analysis of gun barrels. Chen et al. (2007) presented an inverse method for estimation of heat flux in a multi-layer gun barrel at continuous firing conditions which showed that the bore surface material can be melted due to heat flux of shoots. Wu et al. (2008) studied the heat transfer in a $155 \mathrm{~mm}$ mid wall cooled compound gun barrel theoretically and numerically and demonstrated ineffectiveness of natural air cooling and effectiveness of forced mid wall cooling for decreasing temperature of the barrel. Chen and Liu (2008) estimated two-dimensional heat flux in a gun barrel by using finite element schemes and an inverse estimation method, and showed the ability of that method for estimation of time varying heat flux and temperature distribution in the gun chamber. Lee et al. (2009) estimated the time varying heat flux and thermal stresses in a machine gun by an inverse algorithm based on the conjugated gradient method. Mishra et al. (2010) computed the heat transfer to the gun barrel for nine shots by the finite element method and validated the model with experimental measurements. In addition, they found that exponentially decaying heat flux was an accurate model for temperature variation of the gun barrel. Hill and Conner (2012) presented a numerical model of transient heat transfer in machine gun barrels which defined the temperature profile through thickness of the barrel by the finite difference method.

The obtaining an exact temperature distribution is of importance due to the need of exact thermal design of the gun barrel. It can be seen from the literature that there is not any exact analytical model introduced for thermal analysis of the gun barrel. Here, an exact closed 
form solution is introduced for the heat conduction equation in cylindrical coordinates under consecutive inner time dependent surface heat flux by both the Fourier and DPL models. The comparison of estimation is done for the solution, and parametrical study is done to show the ability of finding a solution for the best gun thermal design. The conditions of self-ignition and melting relative to the shooting frequency and the number of shoots can be determined exactly by this model. The closed form solution can be applied for verification of other numerical works in this area.

\section{Mathematical model}

The gun barrel model as an infinite hollow cylinder is shown in Fig. 1 with inner and outer radius of $r_{i}$ and $r_{o}$.

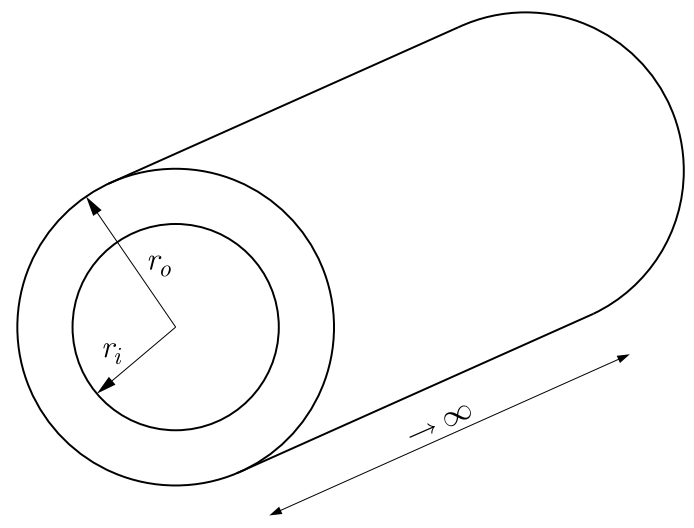

Fig. 1. Schematic of hollow cylindrical model

The one-dimensional DPL thermal wave model in cylindrical coordinate is considered as

$$
\tau_{q} \frac{\partial^{2} T}{\partial t^{2}}+\frac{\partial T}{\partial t}=\alpha\left[\frac{\partial^{2} T}{\partial r^{2}}+\frac{1}{r} \frac{\partial T}{\partial r}+\tau_{T}\left(\frac{1}{r} \frac{\partial^{2} T}{\partial t \partial r}+\frac{\partial}{\partial t} \frac{\partial^{2} T}{\partial r^{2}}\right)\right]
$$

The inner boundary condition of the barrel is considered as subject to an exponentially decaying heat flux as $q\left(r_{i}, t\right)=q_{0} f(t)$ for each shoot, where $f(t)$ is defined as $f(t)=\exp \left(-t / t_{0}\right)$ (Mishra et al., 2010) as shown in Fig. 2 in which $t_{0}$ is a constant that depends on pressure and muzzle velocity and projectile mass (Lawton, 2001). The two time-dependent function of the produced heat flux for Gun (2) (Seiler et al., 2003) and Gun (3) (Dębski et al., 2016) is shown in Fig. 2.

Thus, the time dependent boundary condition on the inner surface is

$$
\tau_{T} \frac{\partial^{2} T}{\partial t \partial r}+\frac{\partial T}{\partial r}=-\frac{q_{0}}{k}\left(f(t)+\tau_{q} \frac{d f(t)}{d t}\right) \quad r=r_{i}
$$

This boundary condition can be seen as a first order ordinary differential equation related to time, which can be solved to get

$$
\left.\frac{\partial T}{\partial r}\right|_{r=r_{i}}=-\frac{q_{0}}{k} \frac{t_{0}-\tau_{q}}{t_{0}-\tau_{T}}\left(\mathrm{e}^{-\frac{t}{t_{0}}}-\mathrm{e}^{-\frac{t}{\tau_{T}}}\right)
$$

The outer surface of the gun barrel is considered to be exposed to air, therefore, undergoes convection heat transfer, which can be defined as

$$
\left.\frac{\partial T}{\partial r}\right|_{r=r_{o}}=h\left(T-T_{\infty}\right)
$$




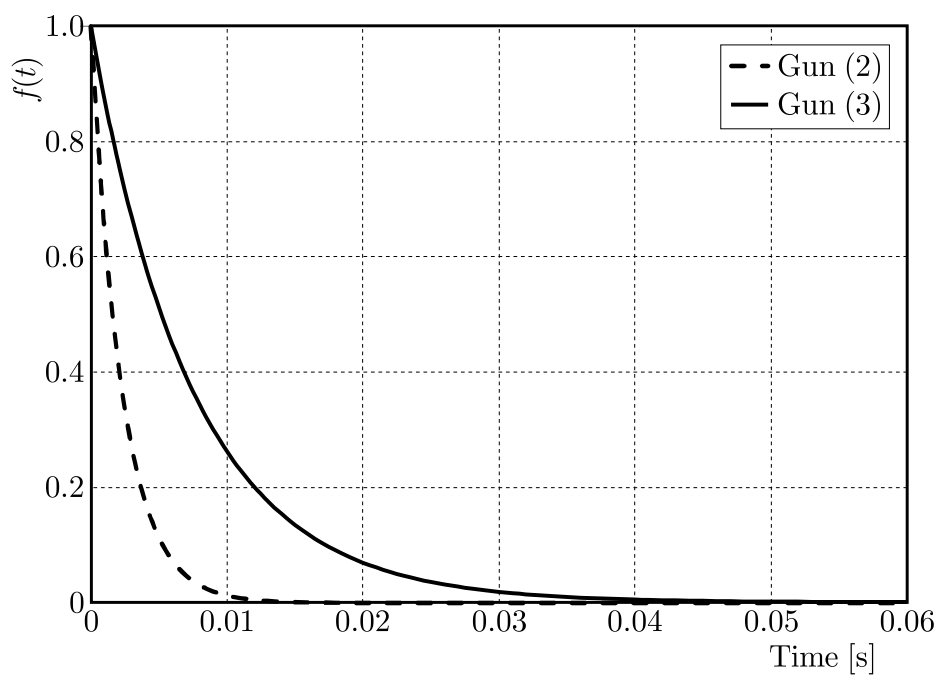

Fig. 2. The time-dependent function of heat flux applied to the inner surface of the gun barrel, $f(t)$

The initial conditions of this problem are

$$
T(r, 0)=\left.T_{\infty} \quad \frac{\partial T}{\partial t}\right|_{t=0}=0
$$

\section{Analytical solution procedure}

The method of separation of variables can not be applied at first due to the time-dependent boundary condition of the inner surface. Therefore, this kind of problem should be solved with a time-independent boundary condition, and then its time dependency should be applied to the solution by using Duhamel's integral. Thus the pure analytical solution procedure according to the superposition principle consists of four steps (Talaee et al., 2014, 2016, 2018; Atefi and Talaee, 2011; Talaee and Sarafrazi, 2017; Talaee and Atefi, 2011; Talaee and Kabiri, 2017a,b):

- The steady equation with a non-homogeneous time-independent boundary condition $\left[\theta_{0}(r)\right]$

- The transient equation with homogeneous and time-independent boundary conditions and the reformed initial condition $\left[\theta_{1}(r, t)\right]$

- Summing the steady and transient solutions as a solution to the problem with time-independent boundary conditions

- Applying the time function of the boundary condition to the final solution using Duhamel's integral.

The above steps are explained in the following. According to the compliance of the results and the solution procedure, the variable $\theta=T-T_{\infty}$ is used afterwards.

\subsection{Steady-state problem}

The steady equation with the non-homogeneous boundary condition is

$$
\frac{d^{2} \theta_{0}}{d r^{2}}+\frac{1}{r} \frac{d \theta_{0}}{d r}=0
$$

and

$$
\left.\frac{d \theta_{0}}{d r}\right|_{r_{i}}=-\left.\frac{q_{0}}{k} \frac{t_{0}-\tau_{q}}{t_{0}-\tau_{T}} \quad \frac{d \theta_{0}}{d r}\right|_{r_{o}}=-\frac{h}{k} \theta_{0}\left(r_{o}\right)
$$


Solution to the steady problem can be simply obtained as

$$
\theta_{0}(r)=\frac{q_{0} r_{i}}{k} \frac{t_{0}-\tau_{q}}{t_{0}-\tau_{T}}\left(\frac{k}{h r_{o}}+\ln \frac{r_{o}}{r}\right)
$$

\subsection{Transient problem}

The transient DPL heat conduction equation with homogeneous boundary conditions and the reformed initial condition is shown in Eqs. (3.4) to (3.6), respectively

$$
\begin{aligned}
& \tau_{q} \frac{\partial^{2} \theta_{1}}{\partial t^{2}}+\frac{\partial \theta_{1}}{\partial t}=\alpha\left(\frac{\partial^{2} \theta_{1}}{\partial r^{2}}+\frac{1}{r} \frac{\partial \theta_{1}}{\partial r}+\tau_{T} \frac{1}{r} \frac{\partial^{2} \theta_{1}}{\partial t \partial r}+\tau_{T} \frac{\partial}{\partial t} \frac{\partial^{2} \theta_{1}}{\partial r^{2}}\right) \\
& \left.\frac{\partial \theta_{1}}{\partial r}\right|_{r_{i}}=\left.0 \quad \frac{\partial \theta_{1}}{\partial r}\right|_{r_{o}}=0 \\
& \theta_{1}(r, 0)=-\left.\theta_{0}(r) \quad \frac{\partial \theta_{1}}{\partial t}\right|_{t=0}=0
\end{aligned}
$$

The solution yields by separating the variables $\left(\theta_{1}(r, t)=R(r) T(t)\right)$ in Eqs. (3.4) to (3.6) as

$$
\theta_{1}(r, t)=\sum_{m=1}^{\infty}\left(A_{m} \mathrm{e}^{r_{1} t}+B_{m} \mathrm{e}^{r_{2} t}\right) \Phi_{m}(r)
$$

where

$$
\begin{aligned}
& r_{1}, r_{2}=\frac{-\left(1+\alpha \lambda_{m}^{2} \tau_{T}\right) \pm \sqrt{\left(1+\alpha \lambda_{m}^{2} \tau_{T}\right)^{2}-4 \tau_{q} \alpha \lambda_{m}^{2}}}{2 \tau_{q}} \\
& \Phi_{m}(r)=J_{0}\left(\lambda_{m} r\right)-\frac{J_{1}\left(\lambda_{m} r_{i}\right)}{Y_{1}\left(\lambda_{m} r_{i}\right)} Y_{0}\left(\lambda_{m} r\right)
\end{aligned}
$$

and $J_{0}, Y_{0}$ are the Bessel functions of the first and second kind, respectively, and $\lambda=\lambda_{m}$ is the root of the following transcendental equation that is obtained by application of boundary conditions

$$
Y_{1}\left(\lambda r_{i}\right)\left(\frac{h}{k} J_{0}\left(\lambda r_{o}\right)-\lambda J_{1}\left(\lambda r_{o}\right)\right)-J_{1}\left(\lambda r_{i}\right)\left(\frac{h}{k} Y_{0}\left(\lambda r_{o}\right)-\lambda Y_{1}\left(\lambda r_{o}\right)\right)=0
$$

Applying the initial conditions in Eq. (3.6), two equations are defined for coefficients $A_{m}$ and $B_{m}$ as

$$
A_{m}+B_{m}=-\frac{\int_{r_{i}}^{r_{o}} \theta_{0}(r) \Phi_{m}(r) r d r}{\int_{r_{i}}^{r_{o}} \Phi_{m}^{2}(r) r d r}=I \quad A_{m} r_{1}+B_{m} r_{2}=0
$$

So

$$
A_{m}=I \frac{r_{2}}{r_{2}-r_{1}} \quad B_{m}=-I \frac{r_{1}}{r_{2}-r_{1}}
$$

in which $I$ is

$$
\begin{aligned}
I= & -\left\{\frac { q _ { 0 } r _ { i } } { k } \frac { t _ { 0 } - \tau _ { q } } { t _ { 0 } - \tau _ { T } } \left[\frac{k r J_{1}\left(\lambda_{m} r\right)}{h r_{o} \lambda_{m}}-\frac{J_{0}\left(\lambda_{m} r\right)-r \lambda_{m} J_{1}\left(\lambda_{m} r\right) \ln \frac{r_{o}}{r}}{\lambda_{m}^{2}}\right.\right. \\
& \left.\left.-\frac{J_{1}\left(\lambda_{m} r_{i}\right)}{Y_{1}\left(\lambda_{m} r_{i}\right)}\left(\frac{k r Y_{1}\left(\lambda_{m} r\right)}{h r_{o} \lambda_{m}}-\frac{Y_{0}\left(\lambda_{m} r\right)-r \lambda_{m} Y_{1}\left(\lambda_{m} r\right) \ln \frac{r_{o}}{r}}{\lambda_{m}^{2}}\right)\right]\right\} /\left\{\frac { r ^ { 2 } } { 2 } \left[J_{0}^{2}\left(\lambda_{m} r\right)\right.\right. \\
& +J_{1}^{2}\left(\lambda_{m} r\right)+\left(\frac{J_{1}\left(\lambda_{m} r_{i}\right)}{Y_{1}\left(\lambda_{m} r_{i}\right)}\right)^{2}\left[Y_{0}^{2}\left(\lambda_{m} r\right)+Y_{1}^{2}\left(\lambda_{m}\right)\right]-2 \frac{J_{1}\left(\lambda_{m} r_{i}\right)}{Y_{1}\left(\lambda_{m} r_{i}\right)}\left[J_{0}\left(\lambda_{m} r\right) Y_{0}\left(\lambda_{m} r\right)\right. \\
& \left.\left.\left.+J_{1}\left(\lambda_{m} r\right) Y_{1}\left(\lambda_{m} r\right)\right]\right]\right\}
\end{aligned}
$$




\subsection{Superposition of the temperature field}

According to the superposition principle, solution of the problem with a time-independent boundary condition is defined as

$$
\theta(r, t)=\theta_{0}(r)+\theta_{1}(r, t)=\frac{q_{0} r_{i}}{k} \frac{t_{0}-\tau_{q}}{t_{0}-\tau_{T}}\left(\frac{k}{h r_{o}}+\ln \frac{r_{o}}{r}\right)+\sum_{m=1}^{\infty} A_{m} G(t) \Phi_{m}(r)
$$

where

$$
G(t)=\mathrm{e}^{r_{1} t}-\frac{r_{1}}{r_{2}} \mathrm{e}^{r_{2} t}
$$

\subsection{Application of Duhamel's integral}

In this step, the complete solution is defined by applying the function $F(t)$ on the solution of the problem with the time-independent boundary condition $\theta(r, t)$. The Duhamel relation can be written as (Talaee and Atefi, 2011)

$$
\bar{\theta}(r, t)=F(0) \theta(r, t)+\int_{0}^{t} \theta(r, t-\tau) \frac{\partial F(\tau)}{\partial \tau} d \tau
$$

Here $F(t)=\mathrm{e}^{-t / t_{0}}-\mathrm{e}^{-t / \tau_{T}}$ is the time-dependent part of the boundary condition, and $F(0)=0$. Thus, the complete solution can be determined as

$$
\bar{\theta}(r, t)=\int_{0}^{t}\left\{\left[\frac{q_{0} r_{i}}{k} \frac{t_{0}-\tau_{q}}{t_{0}-\tau_{T}}\left(\frac{k}{h r_{o}}+\ln \frac{r_{o}}{r}\right)+\sum_{m=1}^{\infty} A_{m} G(t-\tau) \Phi_{m}(r)\right]\left(\frac{\mathrm{e}^{-\frac{\tau}{\tau_{T}}}}{\tau_{T}}-\frac{\mathrm{e}^{-\frac{\tau}{t_{0}}}}{t_{0}}\right) d \tau\right\}
$$

Doing time integration of the above solution, the final closed form solution of the temperature distribution in the gun barrel due to a single shoot is reduced to

$$
\begin{aligned}
& \bar{\theta}(r, t)=\theta_{0}(r)\left(\mathrm{e}^{-\frac{t}{t_{0}}}-\mathrm{e}^{-\frac{t}{\tau_{T}}}\right)+\sum_{m=1}^{\infty} \Phi_{m}(r)\left[A_{m}\left(\frac{\mathrm{e}^{r_{1} t}-\mathrm{e}^{-\frac{t}{\tau_{T}}}}{r_{1} \tau_{T}+1}-\frac{\mathrm{e}^{r_{1} t}-\mathrm{e}^{-\frac{t}{t_{0}}}}{r_{1} t_{0}+1}\right)\right. \\
& \left.+B_{m}\left(\frac{\mathrm{e}^{r_{2} t}-\mathrm{e}^{-\frac{t}{\tau_{T}}}}{r_{2} \tau_{T}+1}-\frac{\mathrm{e}^{r_{2} t}-\mathrm{e}^{-\frac{t}{t_{0}}}}{r_{2} t_{0}+1}\right)\right]
\end{aligned}
$$

For consecutive shooting, $f(t)$ can be a series of single shoots as introduced (Talaee et al., 2018) in Eq. (3.16)

$$
f(t)=\sum_{n=1}^{s} \mathrm{e}^{-\frac{t-(n-1) u}{t_{0}}}
$$

where $u$ is the time interval between the consecutive shoots and $s$ is the number of shoots which is determined as the Bracket function of $[(t / u)+1]$.

Finally, this solution for the consecutive shoots is given as

$$
\begin{aligned}
& \bar{\theta}(r, t)=\theta_{0}(r) \sum_{n=1}^{s}\left(\mathrm{e}^{-\frac{t-(n-1) u}{t_{0}}}-\mathrm{e}^{-\frac{t-(n-1) u}{\tau_{T}}}\right) \\
& +\sum_{n=1}^{s} \sum_{m=1}^{\infty} \Phi_{m}(r)\left[A_{m}\left(\frac{\mathrm{e}^{r_{1}(t-(n-1) u)}-\mathrm{e}^{-\frac{t-(n-1) u}{\tau_{T}}}}{r_{1} \tau_{T}+1}-\frac{\mathrm{e}^{r_{1}(t-(n-1) u)}-\mathrm{e}^{-\frac{t-(n-1) u}{t_{0}}}}{r_{1} t_{0}+1}\right)\right. \\
& \left.+B_{m}\left(\frac{\mathrm{e}^{\left.r_{2}(t-(n-1) u)\right)}-\mathrm{e}^{-\frac{t-(n-1) u}{\tau_{T}}}}{r_{2} \tau_{T}+1}-\frac{\mathrm{e}^{r_{2}(t-(n-1) u)}-\mathrm{e}^{-\frac{t-(n-1) u}{t_{0}}}}{r_{2} t_{0}+1}\right)\right]
\end{aligned}
$$


The solution of the problem for one shoot in the Fourier model, doing the above procedure, is

$$
\bar{\theta}(r, t)=\theta_{0}(r) \mathrm{e}^{-\frac{t}{t_{0}}}+\sum_{m=1}^{\infty} C_{m} \Phi_{m}\left(\mathrm{e}^{-\alpha \lambda_{m}^{2} t}+\frac{\mathrm{e}^{-\alpha \lambda_{m}^{2} t}-\mathrm{e}^{-\frac{t}{t_{0}}}}{\alpha t_{0} \lambda_{m}^{2}-1}\right)
$$

And the solution for the consecutive shoots in the Fourier (parabolic equation) model is

$$
\begin{aligned}
& \bar{\theta}(r, t)=\theta_{0}(r) \sum_{n=1}^{s} \mathrm{e}^{-\frac{t-(n-1) u}{t_{0}}} \\
& +\sum_{n=1}^{s} \sum_{m=1}^{\infty} C_{m} \Phi_{m}\left(\mathrm{e}^{-\alpha \lambda_{m}^{2}(t-(n-1) u)}+\frac{\mathrm{e}^{-\alpha \lambda_{m}^{2}(t-(n-1) u)}-\mathrm{e}^{-\frac{t-(n-1) u}{t_{0}}}}{\alpha t_{0} \lambda_{m}^{2}-1}\right)
\end{aligned}
$$

\section{Results and discussion}

In order to demonstrate the accuracy of the introduced model, the temperature variation during a single shoot is analyzed by both the Fourier and DPL models and compared with experimental data as shown in Fig. 3. The simulation properties are adopted for Gun (1) as discussed in literature in Table 1. As shown in Fig. 4, the dimensionless temperature of both approaches for one shoot have good agreement with the experiment (Lawton, 2001).

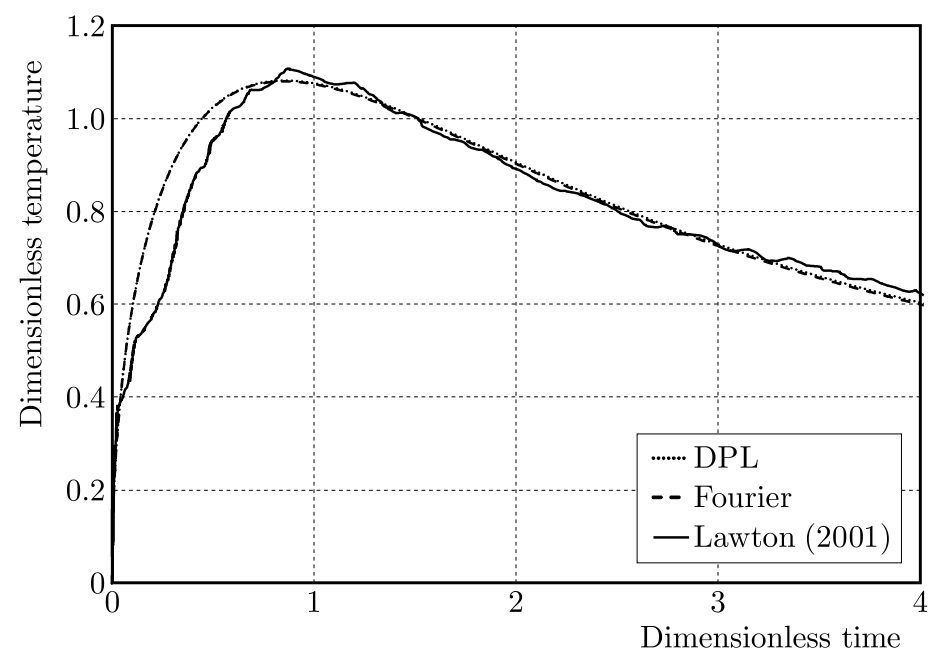

Fig. 3. Dimensionless temperature of the inner surface for a single shoot for Gun (1)

At the next step, the ability of the solution is shown by a comparison with the experiment (Seiler et al., 2003) in the state of consecutive multi shoots for Gun (2) as shown in Fig. 4. The temperature profile is plotted applying real parameters of the guns as shown in Table 1.

The ability of the solution for estimation of temperature distribution is shown in Fig. 4 compared with experimental data.

The temperature distribution in the radial direction of the barrel is shown in Fig. 5. It can be seen that the DPL model has a better estimation than the Fourier one.

The time interval between consecutive shoots or frequency of shooting has an important role in the magnitude of temperature in the barrel. Here, two different time intervals of $0.3 \mathrm{~s}$ and $0.15 \mathrm{~s}$ are adopted for parametrical analysis of temperature profiles using the DPL model. In the other words, the temperature variation at the inner and outer surfaces of the gun barrel are calculated in a specific time for different shooting rates of $200 \mathrm{rpm}$ and $400 \mathrm{rpm}$. 
Table 1. Applied parameters for solving the problem

\begin{tabular}{|c|c|c|c|c|}
\hline \multirow[b]{2}{*}{ Parameters } & \multicolumn{3}{|c|}{ Magnitude } & \multirow[b]{2}{*}{ Dimension } \\
\hline & $\begin{array}{c}\text { Gun (1) (Lawton, } \\
\text { 2001) }\end{array}$ & $\begin{array}{l}\text { Gun (2) (Seiler } \\
\text { et al., 2003) }\end{array}$ & $\begin{array}{c}\text { Gun (3) (Dębski } \\
\text { et al., 2016) }\end{array}$ & \\
\hline $\bar{\alpha}$ & $1.11 \cdot 10^{-5}$ & $1 \cdot 10^{-5}$ & $0.69 \cdot 10^{-5}$ & $\mathrm{~m}^{2} / \mathrm{s}$ \\
\hline$k$ & 40 & 36 & 30 & $\mathrm{w} / \mathrm{mk}$ \\
\hline$\tau_{T}$ & 59 & 59 & 59 & ps \\
\hline$\tau_{q}$ & 0.59 & 0.59 & 0.59 & ps \\
\hline$t_{0}$ & 4.74 & 2.3 & 7.48 & $\mathrm{~ms}$ \\
\hline$q_{0}$ & 192.7 & 272.2 & 262.3 & $\mathrm{Mw} / \mathrm{m}^{2}$ \\
\hline$h_{\infty}$ & 30 & 30 & 30 & $\mathrm{w} /\left(\mathrm{m}^{2} \mathrm{k}\right)$ \\
\hline$T_{\infty}$ & 27 & 27 & 27 & ${ }^{\circ} \mathrm{C}$ \\
\hline $2 r_{i}$ & 155 & 20 & 35 & $\mathrm{~mm}$ \\
\hline Nominal shooting rate & - & 300 & 550 & rounds/min \\
\hline Permeated feed box & - & 20 & - & - \\
\hline
\end{tabular}

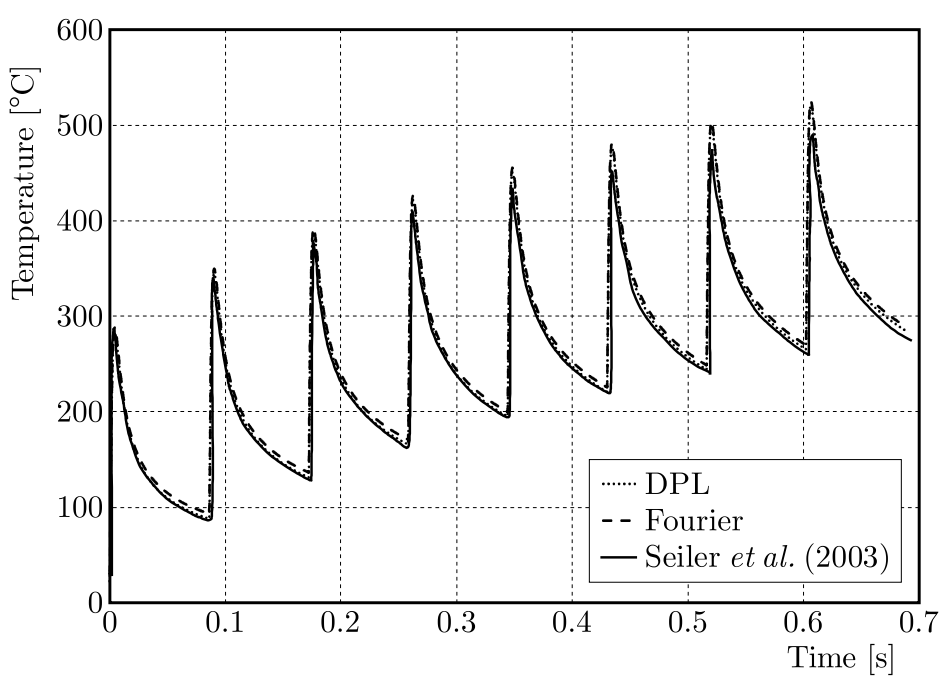

Fig. 4. Temperature of the specified point of the barrel in $150 \mu \mathrm{m}$ from the inner surface for Gun (2) in the consecutive shooting mode

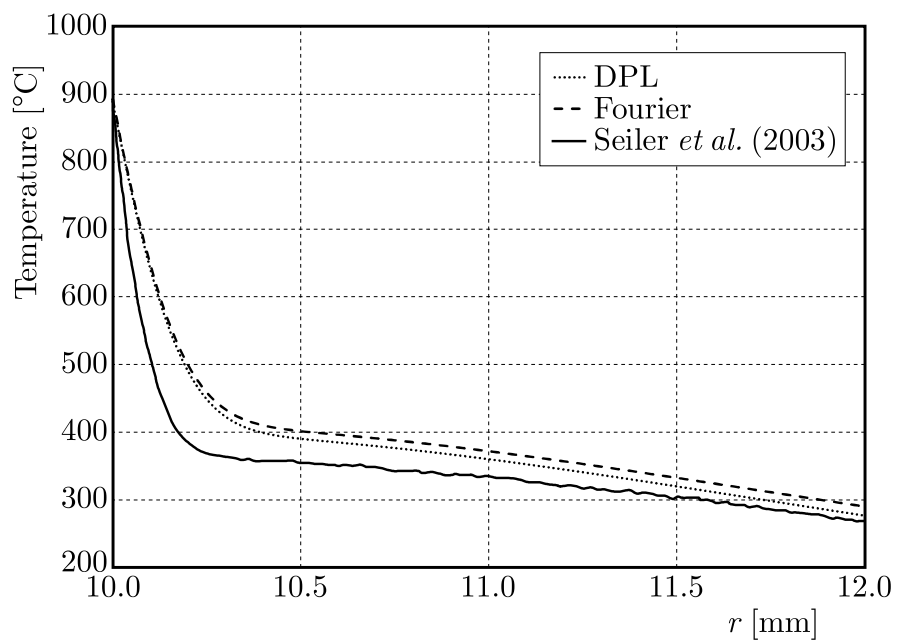

Fig. 5 . Temperature profile at the given time $t=1205.5 \mathrm{~ms}$ at the 15 -th shoot 
The time-history temperature variation of the barrel is analyzed for Gun (2) and (3), and the results are shown below for two types of guns in literature as described in Table 1 . The temperature history of the inner and outer surface of the gun barrel in two shooting rates of $200 \mathrm{rpm}$ and $400 \mathrm{rpm}$ (round per minute) is depicted in Fig. 6, and the temperature profile in barrel thickness is shown in Fig. 7.

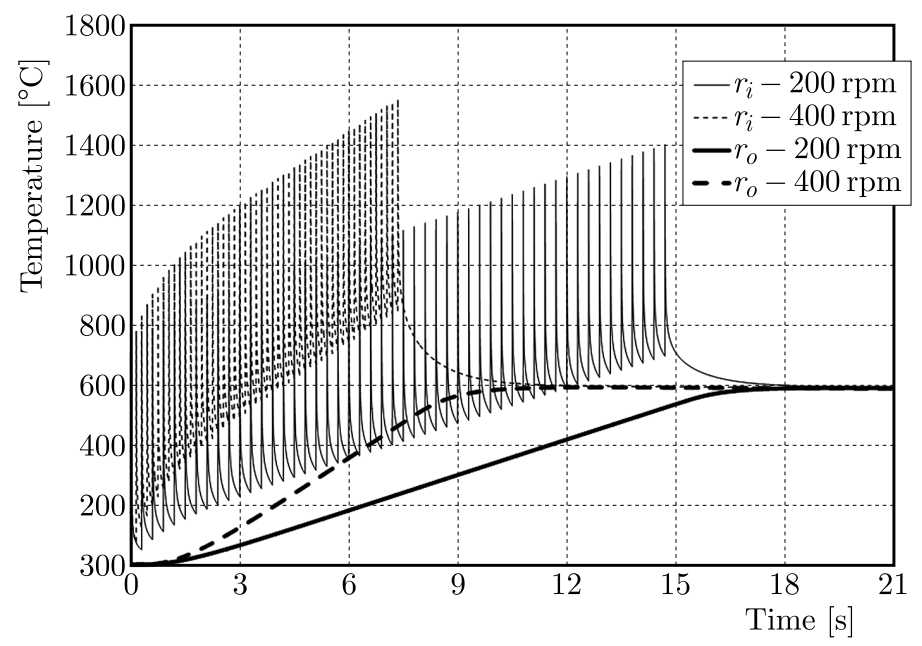

Fig. 6. Temperature variation of the inner and outer surfaces of Gun (2) for consecutive shoots

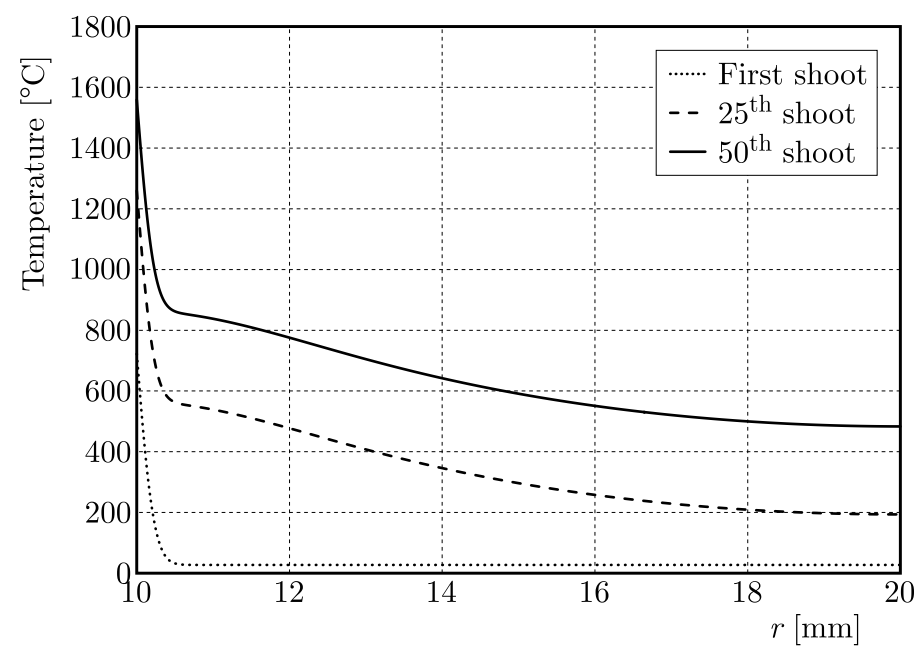

Fig. 7. Temperature distribution in barrel thickness of Gun (2) in $400 \mathrm{rpm}$

As shown in Fig. 7, the rate of temperature rise increases due to the increase of the shooting rate in Gun (2). For example, after about $8 \mathrm{~s}$ or 50 consecutive shoots, the temperature of the inner surface crosses $1560^{\circ} \mathrm{C}$, at the rate of $400 \mathrm{rpm}$, for which the outer surface temperature is about $500^{\circ} \mathrm{C}$. Since the melting point of steel is about $1700^{\circ} \mathrm{C}$, it can be said that the shooting can be continued with the rate of $400 \mathrm{rpm}$ up to 50 shoots. It can be seen in Fig. 7 that the shooting rate or feed box can be increased from the nominal rate of $300 \mathrm{rpm}$ or 20 for this type of gun, respectively.

The temperature behavior is calculated for bigger Gun (3) with higher caliber, as given in Table 1. The results are shown in Figs. 8 and 9.

This gun has a higher caliber and then higher produced heat load on the inner surface compared with Gun (2). Usually, the barrel of large caliber guns can be coated by a hot working material with high melting temperature such as Tantalum (Underwood et al., 2007) which has about $3000^{\circ} \mathrm{C}$ melting temperature (Malter and Langmuir, 1939). As shown in Fig. 8, the tem- 


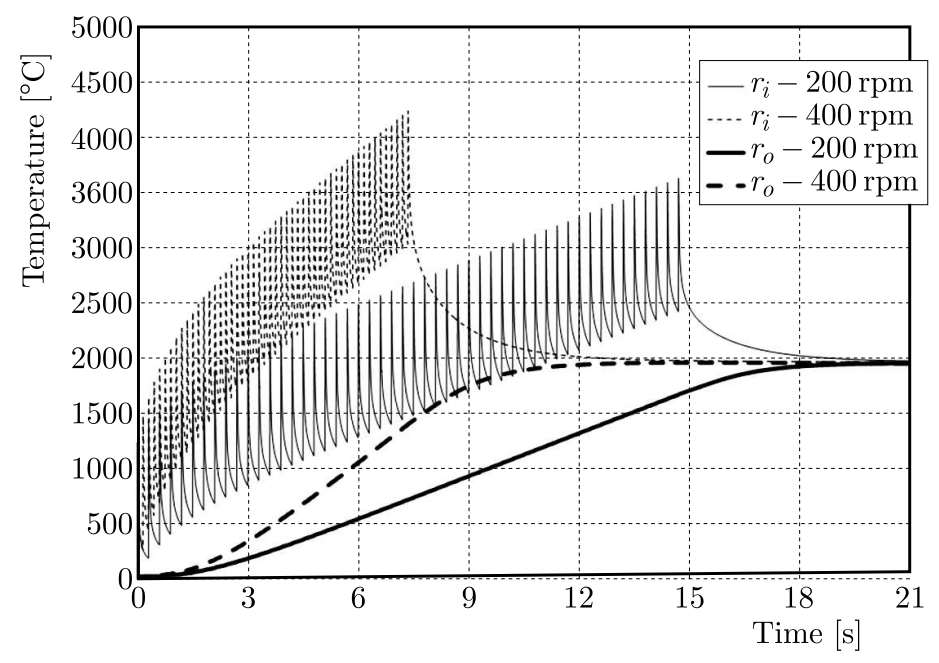

Fig. 8. Temperature variation of the inner and outer surfaces of Gun (3) for consecutive shoots

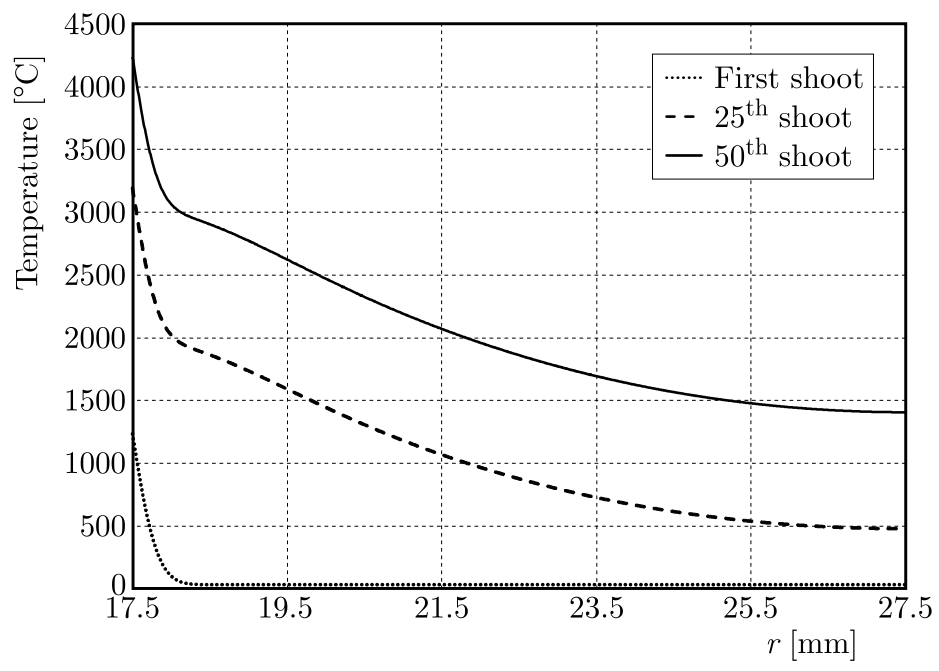

Fig. 9. Temperature distribution in barrel thickness of Gun (3) for $400 \mathrm{rpm}$

perature of the inner and outer surfaces is increased due to an increase in the shooting rate, and after about $3 \mathrm{~s}$ (21 consecutive shoots) the inner surface temperature reaches $3000^{\circ} \mathrm{C}$ at the rate of $400 \mathrm{rpm}$, in which the outer surface temperature is about $350^{\circ} \mathrm{C}$. But at the rate of $200 \mathrm{rpm}$, the inner surface temperature reaches about $2500^{\circ} \mathrm{C}$ after about $6 \mathrm{~s}$ (21 consecutive shoots). Therefore, considering the temperature profile in Fig. 9, it can be said that if this gun is used in the state of $400 \mathrm{rpm}$, some phenomena such as barrel melting and corrosion, decrease of shooting accuracy and self-ignition are probable. It can be said that under consecutive shoots, the thermal conditions are more critical for guns with larger calibers, and unexpected incidents such as barrel melting are so probable. Finally, the ability of the introduced analytical solution for estimation of the temperature profile in the gun barrel is stated as shown above.

\section{Conclusion}

The one-dimensional dual-phase-lag heat conduction in a gun barrel is determined by an analytical solution. The pure analytical solution was found based on the method of separation of variables and Duhamel's integral. The effect of different shooting rates in consecutive shooting and different gun calibers on the magnitude and distribution of temperature has been studied 
in this work. The results show that the decreasing time interval leads to higher temperatures in the gun barrel which may lead to barrel melting and threatens the safety of the shooter. The obtained results can be used for analysis and production of safe gun barrels to prevent from melting, self-ignition and decreasing the shooting accuracy.

\section{References}

1. Afrin N., Zhang Y., Chen J., 2014, Dual-phase lag behavior of a gas-saturated porous-medium heated by a short-pulsed laser, International Journal of Thermal Sciences, 75, 21-27

2. Akbarzadeh A., Chen Z., 2012, Transient heat conduction in a functionally graded cylindrical panel based on the dual phase lag theory, International Journal of Thermophysics, 33, 6, 1100-1125

3. Atefi G., Talaee M.R., 2011, Non-Fourier temperature field in a solid homogeneous finite hollow cylinder, Archive of Applied Mechanics, 81, 5, 569-583

4. Cattaneo C., 1958, A form of heat-conduction equations which eliminates the paradox of instantaneous propagation, Comptes Rendus, 247, 431

5. Chandrasekharaiah D., 1998, Hyperbolic thermoelasticity: a review of recent literature, Applied Mechanics Reviews, 51, 12, 705-729

6. Chen T.-C., Liu C.-C., Jang H.-Y., Tuan P.-C., 2007, Inverse estimation of heat flux and temperature in multi-layer gun barrel, International Journal of Heat and Mass Transfer, 50, 11-12, 2060-2068

7. Chen T.-C., Liu C.-C., 2008, Inverse estimation of time-varied heat flux and temperature on 2-D gun barrel using input estimation method with finite-element scheme, Defence Science Journal, $\mathbf{5 8}, 1,57$

8. Dębski A., Koniorczyk P., Leciejewski Z., Preiskorn M., Surma Z., Zmywaczyk J., 2016, Analysis of heat transfer in a $35 \mathrm{~mm}$ barrel of an anti-aircraft cannon, Problemy Mechatroniki. Uzbrojenie, Lotnictwo, Inżynieria Bezpieczeństwa, 7, 3, 71-86

9. Ghazanfarian J., AbBassi A., 2009, Effect of boundary phonon scattering on dual-phase-lag model to simulate micro- and nano-scale heat conduction, International Journal of Heat and Mass Transfer, 52, 15-16, 3706-3711

10. Ghazanfarian J., Abbassi A., 2012, Investigation of 2D transient heat transfer under the effect of dual-phase-lag model in a nanoscale geometry, International Journal of Thermophysics, 33, 3, $552-566$

11. Ghazanfarian J., Shomali Z., 2012, Investigation of dual-phase-lag heat conduction model in a nanoscale metal-oxide-semiconductor field-effect transistor, International Journal of Heat and Mass Transfer, 55, 21-22, 6231-6237

12. Gheitaghy A., Talaee M., 2013, Solving hyperbolic heat conduction using electrical simulation, Journal of Mechanical Science and Technology, 27, 12, 3885-3891

13. Han P., TANG D., Zhou L., 2006, Numerical analysis of two-dimensional lagging thermal behavior under short-pulse-laser heating on surface, International Journal of Engineering Science, 44, 20, $1510-1519$

14. Hill R.D., Conner J.M., 2012, Transient heat transfer model of machine gun barrels, Materials and Manufacturing Processes, 27, 8, 840-845

15. LaWton B., 2001, Thermo-chemical erosion in gun barrels, Wear, 251, 1-12, 827-838

16. Lee H.-L., Yang Y.-C., Chang W.-J., Wu T.-S., 2009, Estimation of heat flux and thermal stresses in multilayer gun barrel with thermal contact resistance, Applied Mathematics and Computation, 209, 2, 211-221

17. Liu K.-C., Chen H.-T., 2010, Investigation for the dual phase lag behavior of bio-heat transfer, International Journal of Thermal Sciences, 49, 7, 1138-1146 
18. Malter L., Langmuir D., 1939, Resistance, emissivities and melting point of tantalum, Physical Review, 55, 8, 743

19. Mishra A., Hameed A., Lawton B., 2010, A novel scheme for computing gun barrel temperature history and its experimental validation, Journal of Pressure Vessel Technology, 132, 6, 061202

20. Mishra S.C., Sahai H., 2012, Analyses of non-Fourier heat conduction in 1-D cylindrical and spherical geometry - an application of the lattice Boltzmann method, International Journal of Heat and Mass Transfer, 55, 23-24, 7015-7023

21. SAedodin S., Barforoush M., 2017, An exact solution for thermal analysis of a cylindrical object using hyperbolic thermal conduction model, Thermophysics and Aeromechanics, 24, 6, 909-920

22. Seiler F., Mathieu G., Peter H., Zimmermann K., 2003, Experimental and numerical estimation of gun barrel heating for rapid fire, WIT Transactions on Modelling and Simulation, 33

23. Talaee M.R., Alizadeh M., Bakhshandeh S., 2014, An exact analytical solution of non-Fourier thermal stress in cylindrical shell under periodic boundary condition, Engineering Solid Mechanics, 2, 4, 293-302

24. Talaee M.R., Atefi G., 2011, Non-Fourier heat conduction in a finite hollow cylinder with periodic surface heat flux, Archive of Applied Mechanics, 81, 12, 1793-1806

25. Talaee M.R., Kabiri A., 2017a, Analytical solution of hyperbolic bioheat equation in spherical coordinates applied in radiofrequency heating, Journal of Mechanics in Medicine and Biology, 17, 4, 1750072

26. Talaee M.R., Kabiri A., 2017b, Exact analytical solution of bioheat equation subjected to intensive moving heat source, Journal of Mechanics in Medicine and Biology, 17, 5, 1750081

27. Talaee M.R., Kabiri A., Khodarahmi R., 2018, Analytical solution of hyperbolic heat conduction equation in a finite medium under pulsatile heat source, Iranian Journal of Science and Technology, Transactions of Mechanical Engineering, 42, 3, 269-277

28. Talaee M.R., Sarafrazi V., 2017, Analytical solution for three-dimensional hyperbolic heat conduction equation with time-dependent and distributed heat source, Journal of Mechanics, 33, $1,65-75$

29. Talaee M.R., Sarafrazi V., Bakhshandeh S., 2016, Exact analytical hyperbolic temperature profile in a three-dimensional media under pulse surface heat flux, Journal of Mechanics, 32, 3, $339-347$

30. Torabi M., Saedodin S., 2011, Analytical and numerical solutions of hyperbolic heat conduction in cylindrical coordinates, Journal of Thermophysics and Heat Transfer, 25, 2, 239-253

31. Torabi M., Zhang K., 2014, Multi-dimensional dual-phase-lag heat conduction in cylindrical coordinates: Analytical and numerical solutions, International Journal of Heat and Mass Transfer, 78, 960-966

32. Tzou D.Y., 1995, The generalized lagging response in small-scale and high-rate heating, International Journal of Heat and Mass Transfer, 38, 17, 3231-3240

33. Tzou D.Y., 1997, Macro- to Microscale Heat Transfer: The Lagging Behavior, Taylor \& Francis, Washington, DC

34. Underwood J., Vigilante G., Mulligan C., 2007, Review of thermo-mechanical cracking and wear mechanisms in large caliber guns, Wear, 263, 7-12, 1616-1621

35. Vernotte P., 1961, Some possible complications in the phenomena of thermal conduction, Compte Rendus, 252, 2190-2191

36. Wu B., Chen G., Xia W., 2008, Heat transfer in a $155 \mathrm{~mm}$ compound gun barrel with full length integral midwall cooling channels, Applied Thermal Engineering, 28, 8-9, 881-888 